\title{
La réadaptation en paraplégie encourage le retour à la vie active
}

\author{
Xavier Jordan \\ Dr méd., Clinique romande de réadaptation, Sion
}

La réadaptation hautement spécialisée soutient les patients paraplégiques à mobiliser de façon optimale leurs ressources pour récupérer plus d'autonomie. Cela n’améliore pas seulement l'indépendance et la qualité de vie des patients. Le bénéfice économique aussi est important car les patients ont moins recours à des prestations de soins et d'assistance s'ils développent moins de complications et que les rentes versées sont plus faibles grâce à leur activité professionnelle.

La Suisse recense chaque année plus de 250 nouveaux cas de paraplégie. Il s'agit majoritairement de personnes jeunes présentant des lésions de la moelle épinière d'origine accidentelle, souvent après un accident de la circulation ou du travail, mais aussi à la suite d'accidents de sport (parapente, baignade, ski, vélo ou cheval). Un deuxième groupe de patients est nettement plus âgé et souffre d'une paraplégie suite à une maladie, par exemple en raison d'une hernie discale, d'une tumeur ou d'une maladie dégénérative comme la sclérose en plaques.

\section{Les patients paraplégiques doivent faire face à de nombreux défis et complications}

Une paraplégie représente un énorme défi en termes d'adaptation physique et psychique pour chacun mais aussi pour l'entourage. La perte de mobilité et de sensibilité causée par la lésion de la moelle épinière est une entrave importante au quotidien qui bouleverse complètement l'existence.

De plus, la paraplégie entraîne toute une série de complications significatives, mais peu visibles, et qui peuvent parfois menacer le pronostic vital. En plus des troubles des fonctions vésicales, intestinales et sexuelles, l'absence de mobilité et les longues périodes en position couchée ou assise provoquent parfois des ulcères de décubitus qui peuvent contraindre les patients à des séjours hospitaliers de plusieurs semaines. Souvent, ils développent très tôt un tonus musculaire accru qui provoque des postures indésirables et désagréables (spasticité, contractures) et des douleurs qui ne peuvent être maîtrisées qu'à l'aide d'une médication adéquate. Un isolement social et des troubles dépressifs figurent aussi parmi les conséquences fréquentes.

En cas de lésion du système nerveux, d'autres complications, moins connues, surviennent plus fréquemment que dans la population générale: ainsi, la tension artérielle peut être très basse (hypotonie orthostatique) ou augmenter dangereusement (dysréflexie autonome) en raison du dérèglement du système nerveux autonome. Les os s'amincissent en raison de l'inactivité (ostéoporose) et deviennent cassants (fractures) et les articulations moins mobilisées présentent une ossification hétérotopique. Suivant le niveau de la paralysie, des troubles cardiaques et pulmonaires peuvent apparaître très précocement. L'absence d'activité musculaire peut entraîner une thrombose veineuse profonde (TVP) pouvant être à l'origine d'une embolie pulmonaire mortelle. Les problèmes mentionnés restreignent la qualité de vie et encore aujourd'hui l'espérance de vie des personnes concernées. Ils réduisent également l'autonomie et la mobilité et entravent fortement l'inclusion et la participation à la vie sociale et professionnelle [1].

Si les patients sont rapidement transférés dans un centre spécialisé, ils meurent moins souvent et subissent moins de complications, malgré un traitement hospitalier de plus courte durée.

Le progrès médical et les centres spécialisés permettent aujourd'hui d'atteindre de bien meilleurs résultats thérapeutiques.

Dans ce contexte complexe, la prise en charge des personnes paraplégiques dès l'apparition de la paralysie et jusqu'à la fin de la vie est exigeante. Les progrès réalisés 
depuis le début des soins médicaux systématiques pendant la Seconde Guerre mondiale sont impressionnants. L'espérance de vie des paraplégiques s'est nettement allongée entre 1950 et 1980 [2], notamment grâce au développement du cathéter urinaire et donc de la possibilité de vider régulièrement la vessie dans des conditions sûres, ce qui empêche le développent précoce d'une insuffisance rénale aiguë généralement à issue fatale. Des données récentes révèlent une persistance de cette tendance, mais dans une mesure réduite. Des travaux des années 1990 montrent que la prise en charge spécialisée dans des centres pour paraplégiques joue un rôle décisif. Un transfert rapide (c'est-à-dire <30 jours) des personnes atteintes d'une paraplégie d'origine traumatique dans un centre spécialisé a pour conséquence que les patients meurent moins souvent, que leurs complications sont plus rares et moins sévères et que, dans l'ensemble, leurs séjours hospitaliers sont de plus courte durée $[3,4]$. Les soins dans le centre spécialisé sont adaptés aux besoins de ces patients. Dans un premier temps, il s'agit de détecter et de traiter les complications précoces de la paraplégie, ce qui nécessite un personnel qualifié. Dans un deuxième temps, les paraplégiques doivent apprendre à gérer leur corps différent et à effectuer les mesures nécessaires de façon autonome. Ce n'est qu'avec d'importants efforts et beaucoup de patience qu'on peut espérer gagner sur les séquelles de la paralysie, ce qui ne peut s'effectuer que dans un centre spécialisé adapté à leurs besoins. De plus, il s'agit d'éviter des complications tardives en apprenant à chaque paraplégique à en reconnaître les premiers signes et à réagir en conséquence. Cet apprentissage est encadré par un personnel spécialisé.

\section{La réadaptation spécialisée des paraplégiques} facilite le retour dans l'environnement social et réduit les placements dans des institutions de soins.

Les nombreuses répercussions d'une lésion de la moelle épinière sur la santé rendent le suivi médical particulièrement complexe et exigeant. L'OMS a reconnu le caractère hautement spécialisé de la prise en charge des personnes souffrant d'une lésion de la moelle épinière et a souligné les acquis dans les pays à haut revenu, notamment une meilleure survie des patients, plus d'autonomie et un retour à la vie professionnelle [5]. La réadaptation des paraplégiques dans des centres spécialisés facilite leur retour dans leur environnement social ou leurs familles, ce qui réduit le nombre de placements dans des homes ou d'autres institutions de soins [6]. Tous ces progrès ont notamment pu être atteints par le suivi longue durée de ces patients dans des consultations pour paraplé- giques, auprès de spécialistes qui connaissent bien les problèmes de santé spécifiques de ce groupe de patients.

Les complications décrites plus haut sont très fréquentes les premières années et peuvent être létales si elles ne sont pas reconnues à temps et traitées de manière adéquate. Même si l'état de santé se stabilise après cette première phase, des complications plus tardives peuvent survenir progressivement. Le cours de ces complications semble pouvoir être freiné par suivi adéquat. Pour cela, le personnel spécialisé doit contrôler régulièrement les patients, réaliser des examens de dépistage spécifiques, recommander et implémenter les adaptations nécessaires, en accord avec le patient et son entourage. Un suivi à vie dans un

Le suivi à vie permet aux personnes paraplégiques d'occuper et de conserver leur place dans la société.

contexte bien coordonné et interdisciplinaire comme le propose les institutions spécialisées s'avère indispensable [7]. C'est la seule façon d'assurer l'autonomie, la qualité de vie, en maintenant au mieux leur état de santé, afin de permettre à nos concitoyens paraplégiques d'occuper et de conserver leur place dans la société.

L'orientation de la politique sanitaire dans les années à venir sera déterminante pour les patients paraplégiques. Un changement de système tarifaire trop axé sur le court terme pourrait remettre en question la prise en charge holistique telle qu'elle est recommandée par l'OMS et l'ISCoS [8]. Les coûts élevés [9] causés par une paraplégie seront-ils encore couverts? Les efforts des cinquante dernières années, depuis l'ouverture du premier centre pour paraplégiques à Genève en 1963, ont permis d'atteindre un haut niveau de qualité dans le traitement aigu et le suivi à vie des lésés médullaires, s'approchant des objectifs définis par la Convention relative aux droits des personnes handicapées [10] que la Suisse a ratifiée en 2014. Une restriction de l'accès aux soins médicaux spécialisés serait un échec éthique et moral. La pression croissante sur les fournisseurs de prestations pourrait mettre à mal les nombreux efforts consentis pour améliorer l'intégration sociale et la qualité de vie des patients et de leur famille. Elle pourrait aussi être délétère du point de vue économique sur le moyen et long terme, en raison d'une augmentation des coûts en lien avec les complications, la perte de maind'œuvre, et les rentes d'invalidité supplémentaires qui devront être octroyées. 